\title{
On Multilinear Mappings of Nuclear Type
}

\author{
MARIO C. MATOS \\ "Dedicate to the memory of L. NACHBIN"
}

\begin{abstract}
The space of multilinear mappings of nuclear type $\left(s, r_{1}, \ldots, r_{n}\right)$ between Banach spaces is considered, some of its properties are described (including the relationship with tensor products) and its topological dual is characterized as a Banach space of absolutely summing mappings.
\end{abstract}

\section{INTRODUCTION}

In [15] A. Pietsch remarked that the attempt to classify the nonlinear operators between Banach spaces should have its starting point in the study of the different classes of multilinear mappings. See also [4] and [19]. On the other side the research in the Theory of Infinite Dimensional Holomorphy has dealt with several topological vector spaces of $n$-linear mappings. See [1], [3], [5], [6], [7], [8], [10], [11], [12], [13] and [14] among other articles. Motivated by these facts we introduce in this paper the class of $n$-linear mappings of nuclear type $\left(s, r_{1}, \ldots, r_{n}\right)$. We may think them as the multilinear counterpart of the $(n,+\infty, q)$-nuclear linear operators of Pietsch (see [16], Chapter 18) as well as the natural generalization of the $n$-linear mappings of nuclear type considered by $\mathrm{C}$. Gupta in [10]. Naturally we cannot forgive that the nuclear linear

1991 Mathematics Subject Classification: 46E40, 46G20.

Editorial Complutense. Madrid, 1993. 
operators were introduced independently by A. Grothendieck in [9] and A.F. Ruston in [17] and [18]. The spaces we are studying will lead to the consideration of new examples of the holomorphy types conceptually introduced by L. Nachbin in [13] and S. Dineen in [7]. In section 2, after defining the vector space of $n$-linear mappings of nuclear type $\left(s ; r_{1}, \ldots, r_{n}\right)$, we consider a natural complete metrizable topology on it determined by a (quasi-)norm. In the presence of the bounded approximation property, we show that this topology can be generated by a simpler (quasi-)norm when restricted to a convenient dense subspace (that of the finite type $n$ linear mappings). In section 3 we consider certain (quasi-)norms on tensor products and their connections with the spaces of absolutely summing mappings. These results are a preparation for the proofs and results of section 4, which characterize the topological duals of the spaces of multilinear mappings of nuclear type as spaces of absolutely summing mappings. These characterizations might be important for a further study of convolution equations on certain spaces of entire functions on Banach spaces. See remark at the end of this paper.

I would like to thank professors K. Floret and J. Mujica for some remarks and suggestions made during my seminar exposition of these results at UNICAMP.

Now we fix the notations used in this paper. For Banach spaces $E_{1}, \ldots, E_{n}$ and $F$ over $\mathbb{K}(\mathbf{R}$ or $\mathbb{C})$ we denote by $\mathscr{Q}\left(E_{1}, \ldots, E_{n} ; F\right)$ the Banach space of all continuous $n$-linear mappings from $E_{1} \times \ldots \times E_{n}$ into $F$, under the natural norm

$$
\|T\|=\sup _{\substack{x_{k} \in \mathrm{B}_{\mathrm{B}_{1}} \\ k=1, \ldots, n}}\left\|T\left(x_{1}, \ldots, x_{n}\right)\right\| .
$$

Here $\mathrm{B}_{E_{1}}$ denotes the closed unit ball of $E_{k}$ centered at 0 . If $\varphi_{k}$ is in the topological dual $E_{k}$ of $E_{k}, k=1, \ldots, n$ and $b \in F$ we denote by $\varphi_{1} \times \ldots \times \varphi_{n} b$ the element of $\mathscr{L}\left(E_{1}, \ldots, E_{n} ; F\right)$ defined as being $\varphi_{1}\left(x_{1}\right) \ldots \varphi_{n}\left(x_{n}\right) b$ at the point $\left(x_{1}, \ldots, x_{n}\right)$. These mappings generate the vector subspace $\mathscr{L}_{f}\left(E_{1}, \ldots E_{n} ; F\right)$ of the finite type $n$-linear mappings. 
If $s \in(0,+\infty)$ we denote by $\ell_{s}(F)$ (or $\ell_{s}$, if $F=\mathbb{R}$ ) the vector space of all sequences $\left(y_{j}\right)_{j=1}^{\infty}$ of elements of $F$ such that

$$
\left\|\left(y_{j}\right)_{j=1}^{\infty}\right\|_{s}=\left[\sum_{j=1}^{\infty}\left\|y_{j}\right\|^{s}\right]^{\frac{1}{s}}<+\infty
$$

If $s \geq 1,\|\cdot\|_{s}$ is a norm and, for $s<1$, it is an $s$-norm. In any case we have a complete metrizable topological vector space. We denote by $\ell_{s}^{w}(F)$ the vector space of all sequences $\left(y_{j}\right)_{j=1}^{\infty}$ of elements of $F$ such that

$$
\left\|\left(y_{j}\right)_{j=1}^{\infty}\right\|_{\psi, s}=\sup _{\varphi \in B_{F^{\prime}}}\left\|\left(\varphi\left(y_{j}\right)\right)_{j=1}^{\infty}\right\|_{s}<+\infty
$$

$\left(\ell_{s}^{*}(F),\|\cdot\|_{n ; s}\right)$ is a complete metrizable topological vector space. If $s=+\infty$ we consider $\ell_{\infty}(F)=\ell_{\infty}^{w}(F)$ as being the Banach space of all bounded sequences $\left(y_{j}\right)_{j=1}^{\infty}$ of elements of $F$ under the norm

$$
\|\left(y_{j} j_{j=1}^{\infty}\left\|_{\infty}=\right\|\left(y_{j}\right)_{j=1}^{\infty}\left\|_{k, \infty}=\sup _{j \in \mathbf{N}}\right\| y_{j} \|\right.
$$

\section{MULTILINEAR MAPPINGS OF NUCLEAR TYPE $\left(s ; r_{i}, \ldots, r_{n}\right)$}

We consider $s \in(0,+\infty], r_{k} \in[1,+\infty], k=1, \ldots, n$ such that

$$
1 \leq \frac{1}{s}+\frac{1}{r_{1}^{\prime}}+\ldots+\frac{1}{r_{n}^{\prime}}
$$

2.1. Definition. A mapping $T \in \mathscr{L}\left(E_{1}, \ldots, E_{n} ; F\right)$ is said to be nuclear of type $\left(s ; r_{l}, \ldots, r_{n}\right)$ if there are $\left(\lambda_{j}\right)_{j=1}^{\infty} \in \ell_{s}\left(\in c_{0}\right.$ if $\left.s=+\infty\right),\left(y_{j}\right)_{j=1}^{\infty} \in \ell_{\infty}(F)$ and $\left(\varphi_{k j}\right)_{j=1}^{\infty} \in \ell_{r_{i}^{\prime}}^{\prime \prime}\left(E_{k}^{\prime}\right), k=1, \ldots, n$ such that

$$
T\left(x_{1}, \ldots, x_{n}\right)=\sum_{j=1}^{\infty} \lambda_{j} \varphi_{1, j}\left(x_{1}\right) \ldots \varphi_{n j}\left(x_{n}\right) y_{j}
$$


The vector space of all such mappings is denoted by $\mathscr{L}_{\mathrm{N}}^{\left(s ; r_{1}, \ldots, r_{n}\right)}\left(E_{1}, \ldots, E_{n} ; F\right)$ and it is a complete metrizable topological vector space under the $t_{n}$-norm

$$
\|T\|_{N,\left(s, r_{1}, \ldots, r_{s}\right)}=\inf \left\|\left(\lambda_{j}\right)_{j=1}^{\infty}\right\|_{s}\left\|\left(y_{j}\right)_{j=1}^{\infty}\right\|_{\infty} \prod_{k=1}^{n}\left\|\left(\varphi_{k, j}\right)_{j=1}^{\infty}\right\|_{w, r_{t}^{\prime}}
$$

where the infimum is taken over all possible representations of $T$ as described in (1) and $t_{n} \in(0,1]$ is given by

$$
\frac{1}{t_{n}}=\frac{1}{s}+\frac{1}{r_{1}^{\prime}}+\ldots+\frac{1}{r_{n}^{\prime}}
$$

If $r_{1}=\ldots=r_{n}=r$ we replace $\left(s ; r_{1}, \ldots, r_{n}\right)$ by $(s ; r)$ in the preceding notations.

If $t_{n}=1, s$ can be writen in terms of the $r_{k} s$ and we say that $T$ is of nuclear type $\left(r_{1}, \ldots, r_{n}\right)$. In this case $\left(s ; r_{1}, \ldots, r_{n}\right)$ is replaced by $\left(r_{1}, \ldots, r_{n}\right)$ (or $r$, if $r_{1}=\ldots=r_{n}=r$ ) in the above notations. We simplify these notations in the case $r=1$ by ommiting the letter $r$ in the notations.

The following result can be proved easily:

2.2. Proposition, If $T \in \mathscr{I}_{N}^{\left(s, r_{1}, \ldots, r_{-}\right)}\left(E_{l}, \ldots, E_{n} ; F\right), A_{k}$ belongs to $\mathscr{L}\left(D_{k} ; E_{k}\right)$, $k=I, \ldots, n$ and $S \in \mathscr{L}(F ; G)$, then $S \circ T \circ\left(A_{1}, \ldots, A_{n}\right)$ is of nuclear type ( $s$; $\left.r_{l}, \ldots, r_{n}\right)$ and

$$
\left\|S \circ T \circ\left(A_{1}, \ldots, A_{n}\right)\right\|_{N,\left(s, r_{1}, \ldots, r\right.} \leq\|S\|\|T\|_{N,\left(s, r_{1}, \ldots, r_{n},{ }_{k}\right)} \prod_{k=1}^{n}\left\|A_{k}\right\|
$$

Another characterization of the $n$-linear mappings of nuclear type $\left(s ; r_{1}, \ldots, r_{n}\right)$ uses the following examples.

\subsection{Examples.}

(1) It is clear that $\mathscr{L}_{f}\left(E_{1}, \ldots, E_{n} ; F\right) \subset \mathscr{Q}_{N}^{\left(s_{1}, r_{\left.1, \ldots, r_{n}\right)}\right)}\left(E_{1}, \ldots, E_{n} ; F\right)$ and 


$$
\left\|\varphi_{1} \times \ldots \times \varphi_{n} b\right\|_{N,\left(s, r_{1}, \ldots, r_{n}\right)}=\left\|\varphi_{1}\right\| \ldots\left\|\varphi_{n}\right\|\|b\|
$$

for $\varphi_{k} \in E_{k}^{\prime}, k=1, \ldots, n$ and $b \in F$.

(2) An easy application of Hölder's inequality shows that

$$
\|T\| \leq\|T\|_{N_{,}\left(s: r_{1}, \ldots, r_{n}\right)}
$$

for every $T \in \mathscr{L}_{N}^{\left(s ; r_{1}, \ldots, r_{n}\right)}\left(E_{1}, \ldots, E_{n} ; F\right)$.

(3) We consider $\left(\sigma_{j}\right)_{j=1}^{\infty}$ in $\ell_{s}$ for $s \in(0,+\infty)$ and in $c_{0}$ for $s=+\infty$. Now we consider the "diagonal" mapping

$$
D_{\left.(\sigma)_{j}\right)_{1}^{*}} \in \mathscr{L}\left(\ell_{r_{1}} ; \ldots, \ell_{r_{n}} ; \ell_{l}\right)
$$

defined by

$$
D_{\left(\sigma, j_{j=1}^{\infty}\right)}\left(\left(\xi_{1, j}\right)_{j=1}^{\infty}, \ldots,\left(\xi_{n, j}\right)_{j=1}^{\infty}\right)=\left(\sigma \xi_{j, j} \ldots \xi_{n, j}\right)_{j=1}^{\infty}
$$

We note that this mapping can be represented by

$$
D_{\left(\sigma_{j}\right)=1}=\sum_{j=1}^{\infty} \sigma_{j}\left(\pi_{j} \times \ldots \times \pi_{j}\right) e_{j}
$$

where $\pi_{j}\left(\left(\xi_{k, m}\right)_{m=1}^{\infty}\right)=\xi_{k, j}$ for $k=1, \ldots, n$ and $j \in \mathbb{N}$ and $e_{j}=(0, \ldots, 0,1,0 \ldots)$, with 1 in the $j$-th component. Since $\left(\pi_{j}\right)_{j=1}^{\infty} \in \ell_{r_{k}^{\prime}}^{w_{1}^{\prime}}\left(\ell_{r_{k}}\right) \subset \ell_{r_{k}^{\prime}}^{w}\left(\left(\ell_{r_{i}^{\prime}}\right)^{\prime}\right)$ (stric inclusion for $\left.r_{k}^{\prime}=+\infty\right)$ with $\left\|\left(\pi_{j}\right)_{j=1}^{\infty}\right\|_{w, r_{j}^{\prime}}=1$, for $k=1, \ldots, n$ and $\left\|e_{j}\right\|=1$ for all $j \in \mathbb{N}$ we have $D_{(\sigma)_{j-1}^{*}}$ of nuclear type $\left(s ; r_{1}, \ldots, r_{n}\right)$ and

$$
\left\|D_{(\sigma,)_{j-1}^{m}}\right\|_{N,\left(s, r_{1}, \ldots, r_{n}\right.} \leq\left\|\left(\sigma_{j}\right)_{j=1}^{\infty}\right\|_{s}
$$

2.4. Theorem. For $T \in \mathscr{L}\left(E_{1}, \ldots, E_{n} ; F\right)$ the following conditions are equivalent:

(a) $T$ is of nuclear type $\left(s ; r_{1}, \ldots, r_{n}\right)$.

(b) There are $A_{k} \in \mathscr{L}\left(E_{k^{\prime}} ; l_{r_{k}}\right), k=1, \ldots, n, Y \in \mathscr{L}\left(l_{l} ; F\right)$ and $\left(\sigma_{j}\right)_{j=1}^{\infty} \in$ $\ell_{x}\left(\in c_{0}\right.$ when $\left.s=+\infty\right)$ such that 


$$
T=Y \circ D_{\left(\sigma_{j}\right)_{1-1}^{-}} \circ\left(A_{1}, \ldots, A_{k}\right)
$$

In this case

$$
\|T\|_{N,\left(s, r_{1}, \ldots, r_{n}\right)}=\inf \|Y\| \prod_{k=1}^{n}\left\|A_{k}\right\|\left\|\left(\sigma_{j}\right)_{j=1}^{\infty}\right\|_{s}
$$

where the infimum is taken over all possible factorizations as decribed in (b).

Proof. By 2.2 and 2.3 (3) we have that (b) implies (a) with

$$
\|T\|_{N,\left(s, r_{1}, \ldots, r_{n}\right)} \leq\|Y\| \prod_{k=1}^{n}\left\|A_{k}\right\| \cdot\left\|\left(\sigma_{j}\right)_{j=1}^{\infty}\right\|_{s}
$$

Now we assume (a). For each $\varepsilon>0$ we consider a representation of $T$ as in 2.1. such that

$$
\left\|\left(\lambda_{j}\right)_{j=1}^{\infty}\right\|_{s}\left\|\left(y_{j}\right)_{j=1}^{\infty}\right\|_{\infty} \prod_{k=1}^{n}\left\|\left(\varphi_{k j}\right)_{j=1}^{\infty}\right\|_{w, r_{k}} \leq(1+\varepsilon)\|T\|_{N,\left(s, r_{1}, \ldots, r_{n}\right)}
$$

If we define $A_{k}\left(x_{k}\right)=\left(\varphi_{k_{j}}\left(x_{k}\right)\right)_{j=1}^{\infty}$ for $x_{k} \in E_{k}$, then $A_{k} \in \mathscr{L}\left(E_{k^{\prime}} l_{r^{\prime}}\right)$ with $\left\|A_{k}\right\| \leq\left\|\left(\varphi_{k j}\right)_{j=1}^{\infty}\right\|_{w, r_{k}^{\prime}}, k=1, \ldots, n$. Now we consider $Y \in \mathscr{L}\left(\ell_{1} ; F\right)$ defined by

$$
Y\left(\left(\xi_{j}\right)_{j=1}^{\infty}\right)=\sum_{j=1}^{\infty} \xi_{j} b_{j}
$$

for $\left(\xi_{j}\right)_{j=1}^{\infty} \in \ell_{1}$. Thus $\|Y\| \leq\left\|\left(b_{j}\right)_{j=1}^{\infty}\right\|_{\infty}$. If follows that

$$
T=Y \circ D_{\left(\lambda_{j}\right) ;-1=1} \circ\left(A_{1}, \ldots, A_{n}\right)
$$

with 


$$
\|Y\|\left\|\left(\lambda_{j}\right)_{j=1}^{\infty}\right\|_{s} \prod_{k=1}^{n}\left\|A_{k}\right\| \leq(1+\varepsilon)\|T\|_{N,\left(s, r_{1}, \ldots, r_{n}\right)}
$$

Now we consider some inclusion results.

2.5. Theorem. For $s, t \in(0,+\infty], r_{k} p_{k} \in[1,+\infty]$ such that $s \leq t$, $r_{k} \leq p_{k}, k=1, \ldots, n$

$$
1 \leq \frac{1}{s}+\frac{1}{r_{1}^{\prime}}+\ldots+\frac{1}{r_{n}^{\prime}}, 1 \leq \frac{1}{t}+\frac{1}{p_{1}^{\prime}}+\ldots+\frac{1}{p_{n}^{\prime}}
$$

and

$$
\frac{1}{r_{1}}+\ldots+\frac{1}{r_{n}}-\frac{1}{s} \leq \frac{1}{p_{1}}+\ldots+\frac{1}{p_{n}}-\frac{1}{t}
$$

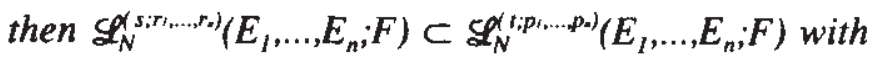

$$
\|T\|_{N,\left(r_{1}, \ldots, \ldots, p_{n}\right)} \leq\|T\|_{N,\left(s ; r_{1}, \ldots, r_{2}\right)}
$$

for every $T$ of nuclear type $\left(s, r_{l}, \ldots, r_{n}\right)$.

Proof. We consider

$$
\frac{1}{q_{k}}=\frac{1}{r_{k}}-\frac{1}{p_{k}}, k=1, \ldots, n ; \frac{1}{u}=\frac{1}{s}-\left(\frac{1}{q_{1}}+\ldots+\frac{1}{q_{n}}\right)
$$

Hence $u \leq t$. For $T$ of nuclear type $\left(s ; r_{1}, \ldots, r_{n}\right)$ and $\varepsilon>0$ we choose a representation of $T$ in the form 


$$
T=\sum_{j=1}^{\infty} \sigma_{j} \varphi_{1, j} \times \ldots \times \varphi_{n, j} y_{j}
$$

such that $\sigma_{j} \geq 0$ for all $j \in \mathbb{N}$ and

$$
\left\|\left(\sigma_{j}\right)_{j=1}^{\infty}\right\|_{s} \prod_{k=1}^{n}\left\|\left(\varphi_{k, j}\right)_{j=1}^{\infty}\right\|_{w, r_{k}}\left\|\left(y_{j}\right)_{j=1}^{\infty}\right\|_{\infty} \leq(1+\varepsilon)\|T\|_{N\left(s, r_{1}, \ldots, r_{n}\right)}
$$

We can write

$$
T=\sum_{j=1}^{\infty} \sigma_{j}^{\frac{s}{u}}\left(\sigma_{j}^{\frac{s}{q_{h}}} \varphi_{1, j}\right) \times \ldots \times\left(\sigma_{j}^{\frac{s}{q_{n}}} \varphi_{n j}\right) y_{j}
$$

and have

$$
\begin{gathered}
\left\|\left(\sigma_{j}^{\frac{s}{u}}\right)_{j=1}^{\infty}\right\|_{t} \leq\left\|\left(\sigma_{j}^{\frac{s}{u}}\right)_{j=1}^{\infty}\right\|_{u}=\left[\left\|\left(\sigma_{j}\right)_{j=1}^{\infty}\right\|_{s}\right]^{\frac{s}{u}} \\
\|\left(\sigma_{j}^{\frac{s}{q_{i}}}\left(\varphi_{k_{j}}\right)_{j=1}^{\infty}\left\|_{w^{\prime}, p_{i}^{\prime}} \leq\right\|\left(\sigma_{j}^{\frac{s}{q_{k}}}\right)_{j=1}^{\infty}\left\|_{q_{k}}\right\|\left(\varphi_{k_{j}}\right)_{j=1}^{\infty} \|_{w^{\prime}, r_{i}^{\prime}}\right. \\
=\left[\left(\sigma_{j}\right)_{j=1}^{\infty} \|_{s}\right]^{\frac{s}{a_{k}}}\left\|\left(\varphi_{k_{j}}\right)_{j=1}^{\infty}\right\|_{w^{\prime}, r_{i}^{\prime}}
\end{gathered}
$$

for $k=1, \ldots, n$. Thus $T$ is of nuclear type $\left(t ; p_{1}, \ldots, p_{n}\right)$ and

$$
\|T\|_{N,\left(r, p_{1}, \ldots, p_{n}\right)} \leq(\mathbb{1}+\varepsilon)\|T\|_{N,\left(s ; r_{1}, \ldots, r_{n}\right)}
$$

for each $\varepsilon>0$.

2.6. Corollary. (1) If $r_{k} \leq p_{k}, k=1, \ldots, n$ every $T$ of nuclear type $\left(r_{l}, \ldots, r_{n}\right)$ is of nuclear type $\left(p_{l}, \ldots, p_{n}\right)$ and 


$$
\|T\|_{N,\left(p_{1}, \ldots, p_{n}\right)} \leq\|T\|_{N,\left(r_{1}, \ldots, r_{n}\right)}
$$

(2) If $s \leq t$, every $T$ of nuclear type $\left(s ; r_{I}, \ldots, r_{n}\right)$ is of nuclear type $\left(t, r_{1}, \ldots, r_{n}\right)$ and

$$
\|T\|_{N_{1}\left(t ; r_{1}, \ldots, r_{n}\right)} \leq\|T\|_{N_{1}\left(s ; r_{1}, \ldots, r_{n}\right)}
$$

(3) If $r_{k} \geq p_{k}, k=1, \ldots, n$ every $T$ of nuclear type $\left(s ; r_{1}, \ldots, r_{n}\right)$ is of nuclear type $\left(s ; p_{1} ; \ldots, p_{n}\right)$ and

$$
\|T\|_{s,\left(r p_{1}, \ldots, p_{x}\right)} \leq\|T\|_{N_{1}\left(s: r_{1}, \ldots, r_{n}\right)}
$$

2.7. Remark. It follows from the definition that $\mathscr{L}_{f}\left(E_{1}, \ldots, E_{n} ; F\right)$ is dense in $\mathscr{L}_{N}^{(s ; n, \ldots, r)}\left(E_{1}, \ldots, E_{n} ; F\right)$. Since every $T$ of $\mathscr{L}_{d}\left(E_{1}, \ldots, E_{n} ; F\right)$ has a finite representation of the form

$$
T=\sum_{j=1}^{m} \sigma_{j} \varphi_{1, j} \times \ldots \times \varphi_{n, j} b_{j}
$$

with $\sigma_{j} \in \mathbb{K}, \varphi_{k, j} \in E_{k}^{\prime}, k=1, \ldots, n, b_{j} \in F, j=1, \ldots, m$, it is natural to ask when it is possible to have

$$
\|T\|_{N_{\left(, s ; r_{r} ; \ldots, r_{s}\right)}}=\|T\|_{N_{f}\left(s ; r_{1}, \ldots, r_{n}\right)}
$$

where

$$
\left.\|T\|_{N_{\rho}\left(s, r_{1}, \ldots, r_{\mu}\right)}=\inf \left\|\left(\sigma_{j}\right)_{j=1}^{m}\right\|_{s} \prod_{k=1}^{n}\left\|\left(\varphi_{k, j}\right)_{j=1}^{m}\right\|_{w, r_{i}} \|(b)_{j}^{m}\right)_{j=1}^{m} \|_{\infty}
$$

with the infimum taken for all representations of $T$ as in (2). It is clear that we always have

$$
\|T\|_{N,\left(s ; r_{1}, \ldots, r_{n}\right)} \leq\|T\|_{N_{f}\left(s ; r_{1}, \ldots, r_{n}\right)}
$$

It is natural to hope that equality might take place when $E_{k}$ is finite 
dimensional for $k=1, \ldots, n$. This is indead a fact as we show in the following result.

2.8. Proposition. If $E_{1}, \ldots, E_{n}$ are finite dimensional and $T \in$ $\mathscr{L}\left(E_{l}, \ldots, E_{n} ; F\right)$, then

$$
\|T\|_{N_{\mu}\left(s, r_{1}, \ldots, r_{n}\right)}=\|T\|_{N,\left(s ; r_{1}, \ldots, r_{n}\right)}
$$

Proof. In this case $\mathscr{L}\left(E_{1}, \ldots, E_{n} ; F\right)=\mathscr{L}_{f}\left(E_{1}, \ldots, E_{n} ; F\right)$ and this is a complete space for both $t_{n}$-norms $\|\cdot\|_{N,\left(s ; r, \ldots, \ldots, r_{n}\right)}$ and $\|\cdot\|_{N_{f}\left(s ; r, \ldots, \ldots, r_{0}\right)}$. By the open mapping theorem these $t_{n}$-norms are equivalent. Hence there is $c \geq 0$ such that

$$
\|T\|_{N_{r}\left(s, r_{i}, \ldots, r_{r}\right.} \leq c\|T\|_{N,\left(s ; r_{1}, \ldots, r_{n}\right)}
$$

for every $T$ of finite type. For $\varepsilon>0$ we choose a representation

$$
T=\sum_{j=1}^{\infty} \sigma_{j} \varphi_{1, j} \times \ldots \times \varphi_{n j} y_{j}
$$

such that

$$
\left\|\left(\sigma_{j}\right)_{j=1}^{\infty}\right\|_{s}\left\|\left(y_{j}\right)_{j=1}^{\infty}\right\|_{\infty} \prod_{k=1}^{n}\left\|\left(\varphi_{k, j}\right)_{j=1}^{\infty}\right\|_{n, r_{k}} \leq(1+\varepsilon)\|T\|_{N,\left(s, r_{1}, \ldots, r_{n}\right)}
$$

There is $m \in \mathbb{N}$ such that

$$
c\left\|\sum_{j>m} \sigma_{j} \varphi_{1, j} \times \ldots \times \varphi_{n j} y_{j}\right\|_{N_{1}\left(s ; r_{1}, \ldots, r_{a}\right)} \leq \varepsilon\|T\|_{N,\left(s, r_{1}, \ldots, r_{n}\right)}
$$

We have 


$$
\begin{aligned}
& \left(\|T\|_{N_{f}\left(s, r_{1}, \ldots, r_{n}\right.}\right)^{t_{n} \leq} \leq\left(\left\|\sum_{j=1}^{m} \sigma_{j} \varphi_{1_{j}} \times \ldots \times \varphi_{n_{j}} y_{j}\right\|_{N_{f}\left(s ; r_{1}, \ldots, r_{n}\right)}\right)^{t_{n}} \\
& +\left(\left\|\sum_{j>m} \sigma_{j} \varphi_{1, j} \times \ldots \times \varphi_{n, j} y_{j}\right\|_{N_{f}\left(s ; r_{1}, \ldots, r_{n}\right.}\right)^{f_{n}} \\
& \leq(1+\varepsilon)^{f_{n}}\left(\|T\|_{N,\left(s, r_{1}, \ldots, r_{n}\right)}\right)^{\prime *} \\
& +c^{t_{r}}\left(\left\|\sum_{j>m} \sigma_{j} \varphi_{l_{j}} \times \ldots \times \varphi_{n, j} y_{j}\right\|_{N_{f}\left(s, r_{j}, \ldots, r_{n}\right.}\right)^{t_{n}} \\
& \leq\left[(1+\varepsilon)^{t_{n}}+\varepsilon^{t_{n}}\right]\left(\|T\|_{N,\left(s ; r_{1}, \ldots, r_{n}\right)}\right)^{t_{n}}
\end{aligned}
$$

2.9. Proposition. If $T \in \mathscr{S}_{N}^{\left(s ; r_{1}, \ldots, r_{n}\right)}\left(E_{l}, \ldots, E_{n} ; F\right)$ and $S_{k}$ is in $\mathscr{L}_{f}\left(D_{k} ; E_{k}\right)$, $k=1, \ldots, n$, then

$$
\left\|T \circ\left(S_{1}, \ldots, S_{n}\right)\right\|_{N_{f}\left(s, r_{1}, \ldots, r_{n}\right)} \leq\|T\|_{N_{(}\left(s, r_{1}, \ldots, r_{n}\right)} \prod_{k=1}^{n}\left\|S_{k}\right\|
$$

Proof. If $J_{k}$ is the natural injection from $S_{k}\left(D_{k}\right)$ into $E_{k}$, we can write $S_{k}=J_{k} \circ \tilde{S}_{k}$ with $\left\|\tilde{S}_{k}\right\|=\left\|S_{k}\right\|, k=1, \ldots, n$. Hence $T \circ\left(J_{1}, \ldots, J_{n}\right)$ is in $\mathscr{L}_{f}\left(S_{1}\left(D_{1}\right), \ldots, S_{n}\left(D_{n}\right) ; F\right)$. Now we apply 2.8 . and 2.2 . in order to have the result.

2.10. Theorem. If $E_{I}^{\prime}, \ldots, E_{n}^{\prime}$ have the bounded approximation property, then 


$$
\|T\|_{N_{S^{\prime}\left(s, r_{1}, \ldots, r_{n}\right)}}=\|T\|_{N,\left(s, r_{1}, \ldots, r_{n}\right\}}
$$

for every $T \in \mathscr{L}_{(}\left(E_{l}, \ldots, E_{n} ; F\right)$.

Proof. First we consider $n=2$. The proof for other $n$ 's is analogous as one can note easily. Since $T_{1} \in \mathscr{L}_{f}\left(E_{1} ; \mathscr{L}\left(E_{2} ; F\right)\right)$ for $T_{1}\left(x_{1}\right)\left(x_{2}\right)=T\left(x_{1}, x_{2}\right)$, $x_{k} \in E_{k}, k=1,2$, for each $\varepsilon>0$ there is $S_{1} \in \mathscr{L}_{\lambda}\left(E_{1} ; E_{1}\right)$ such that $T_{1} \circ S_{1}$ $=T_{1}$ and $\left\|S_{1}\right\| \leq(1+\varepsilon) \lambda_{1}$ (because $E_{1}^{\prime}$ has the $\lambda_{1}$-approximation property for some $\left.\lambda_{1}>0\right)$. Hence

$$
T\left(S_{1}\left(x_{1}\right), x_{2}\right)=T\left(x_{1}, x_{2}\right) \quad\left(\forall x_{k} \in E_{k}, k=1,2\right)
$$

By the same reasoning $T_{2} \in \mathscr{L}_{f}\left(E_{2} ; \mathscr{L}\left(E_{1} ; F\right)\right.$ ), given by $T_{2}\left(x_{2}\right)\left(x_{1}\right)=T\left(x_{1}, x_{2}\right)$, with $x_{k} \in E_{k}, k=1,2$, is such that there is $S_{2} \in \mathscr{L}_{j}\left(E_{2} ; E_{2}\right)$ satisfying $T_{2}$ 。 $S_{2}=T_{2}$ and $\left\|S_{2}\right\| \leq(1+\varepsilon) \lambda_{2}$. We have

$$
T\left(x_{1}, S_{2}\left(x_{2}\right)\right)=T\left(x_{1}, x_{2}\right) \quad\left(\forall x_{k} \in E_{k}, k=1,2\right)
$$

Thus $T=T \circ\left(S_{1}, S_{2}\right)$ and, by 2.9 ,

$$
\begin{aligned}
& \|T\|_{N_{f}\left(s ; r_{1}, r_{2}\right)}=\left\|T \circ\left(S_{1}, S_{2}\right)\right\|_{N_{f}\left(s ; r_{1}, r_{2}\right)} \\
& \leq\|T\|_{N_{,\left(s: r_{1}, r_{2}\right)}}\left\|S_{1}\right\|\left\|S_{2}\right\| \leq(1+\varepsilon)^{2} \lambda_{1} \lambda_{2}\|T\|_{N,\left(s: r_{1}, r_{2}\right)}
\end{aligned}
$$

Therefore

$$
\|T\|_{N_{P_{r}\left(s, r_{r}, r_{2}\right)}} \leq \lambda_{1} \lambda_{2}\|T\|_{N,\left(s, r_{1}, r_{2}\right)}
$$

Now with the same argument used in the proof of 2.8 we have

$$
\|T\|_{N_{r}\left(s, r_{1}, r_{2}\right)} \leq\|T\|_{N,\left(s ; r_{r}, r_{2}\right)}
$$




\section{ABSOLUTELY SUMMING MULTILINEAR MAPPINGS AND TENSOR PRODUCTS}

We recall the concept of absolutely $\left(s ; r_{1}, \ldots, r_{n}\right)$-summing $n$-linear mapping from $E_{1} \times \ldots \times E_{n}$ into $F$ (See Pietsch [14] for scalar valued mappings).

In this section, $s, r_{k} \in(0,+\infty], k=1, \ldots, n$ are such that

$$
\frac{1}{s} \leq \frac{1}{r_{1}}+\ldots+\frac{1}{r_{n}}
$$

3.1. Definition. $A$ mapping $T \in \mathscr{L}\left(E_{1}, \ldots, E_{n} ; F\right)$ is said to be absolutely $\left(s, r_{1}, \ldots, r_{n}\right)$-summing if there is $c \geq 0$ such that

$$
\left\|\left(T\left(x_{1, j}, \ldots, x_{n_{j}}\right)\right)_{j=1}^{m}\right\|_{s} \leq c \prod_{k=1}^{n}\left\|\left(x_{k_{j}}\right)_{j=1}^{m}\right\|_{w, r_{t}}
$$

for $m \in \mathbb{N}, x_{k j} \in E_{k}, k=1, \ldots, n$ and $j=1, \ldots, m$.

We denote by $\mathscr{L}_{a s}^{\left(s ; r, \ldots, r_{n}\right)}\left(E_{1}, \ldots, E_{n} ; F\right)$ the vector space of all these mappings. The smallest possible value of $c$ in (3) is denoted by $\|\mathrm{T}\|_{a s,(s, r, \ldots, \ldots, j)}$ This gives a $s$-norm (for $s \in(0,1)$ ) and a norm (for $s \geq 1$ ) making $\mathscr{Q}_{a s}^{\left(s ; r_{1}, \ldots, r_{1}\right)}\left(E_{1}, \ldots, E_{n} ; F\right)$ a complete metrizable topological vector space. When $r_{1}=\ldots=r_{n}=r$ we replace $\left(s ; r_{1}, \ldots, r_{n}\right)$ by $(s ; r)$ in the preceding notations. In the last case for $s=r$ we replace $(s ; r)$ by $r$ in the above notations. When $r=1$ we do not write it.

An interesting special case of absolutely $\left(s ; r_{1}, \ldots, r_{n}\right)$-summing mappings is obtained when we have

$$
\frac{1}{s}=\frac{1}{r_{1}}+\ldots+\frac{1}{r_{n}}
$$

In this situation the mappings are called $\left(r_{1}, \ldots, r_{n}\right)$-dominated and we denote the corresponding vector space and $s$-norm (or norm) by 
$\mathcal{L}_{d}^{\left(r_{1}, \ldots, r_{n}\right)}\left(E_{1}, \ldots, E_{n} ; F\right)$ and \|\|$_{d,\left(r_{1}, \ldots, r_{n}\right)}$ respectively. If $r_{1}=\ldots=r$ we replace $\left(r_{1}, \ldots, r_{n}\right)$ by $r$ in the preceding notations. When $r=1$ we do not write it. The use of the word "dominated" is justified by the following result mentioned by Pietsch in [14], for scalar valued mappings.

3.2. Theorem. For $T \in \mathscr{L}\left(E_{1}, \ldots, E_{n} ; F\right)$ and $s, r_{k} \in(0,+\infty], k=I, \ldots, n$ such that

$$
\frac{1}{s}=\frac{1}{r_{1}}+\ldots+\frac{1}{r_{n}}
$$

the following conditions are equivalent

(a) $T$ is $\left(r_{1}, \ldots, r_{n}\right)$-dominated

(b) There are $c \geq 0$ and regular probability measures $\mu_{k} \in W\left(B_{E_{1}^{\prime}}\right)$, $k=1, \ldots, n$ such that

$$
\left\|T\left(x_{1}, \ldots, x_{n}\right)\right\| \leq c \prod_{k=1}^{n}\left[\int_{B_{\varepsilon_{k}^{\prime}}}\left|\varphi\left(x_{k}\right)\right|^{r_{i}} d \mu_{k}(\varphi)\right]^{\frac{1}{r_{4}}}
$$

for ever $x_{k} \in E_{k}, k=I, \ldots, n$.

In this case

$$
\underset{(4)}{\inf } c=\min _{(4)} c=\|T\|_{d\left(t, \ldots, \ldots, r_{n}\right)}
$$

Here $W\left(B_{E^{\prime}}\right)$ denotes the set of all regular probability measures defined on the Borel $\sigma$-algebra of $B_{E_{x}^{\prime}}$, with the weak-star topology.

Now we are going to introduce some special $t_{n}$-norms on $E_{1} \otimes \ldots \otimes$ $E_{n} \otimes F$ in such way that the topological dual of this metrizable topological vector space is isometric to a space of $F^{\prime}$-valued absolutely summing mappings on $E_{1} \times \ldots \times E_{n}$. 
3.3. Definition. For $r_{k} \in(0,+\infty], k=1, \ldots, n$ and $s \in[1,+\infty]$ such that

$$
\frac{1}{s} \leq \frac{1}{r_{1}}+\ldots+\frac{1}{r_{n}}
$$

we consider $t_{n} \in(0,1]$ such that

$$
\frac{1}{t_{n}}=\frac{1}{r_{1}}+\ldots+\frac{1}{r_{n}}+\frac{1}{s^{\prime}} \geq 1
$$

and define

$$
\delta_{\left(s, r_{1}, \ldots, r_{n_{n}+\infty}\right)}(u)=\inf \left\|\left(\lambda_{j}\right)_{j=1}^{m}\right\|_{s^{\prime}} \prod_{k=1}^{n}\left\|\left(x_{k j}\right)_{j=1}^{m}\right\|_{w, r_{k}}\left\|\left(b_{j}\right)_{j=1}^{m}\right\|_{\infty}
$$

where $u \in E_{l} \otimes \ldots \otimes E_{n} \otimes F$ and the infimum is taken over all representations of $u$ of the form

$$
u=\sum_{j=1}^{m} \lambda_{j} x_{1, j} \otimes \ldots \otimes x_{n, j} \otimes b_{j}
$$

with $m \in \mathbb{N}, x_{k_{i}} \in E_{k}, k=1, \ldots, n, \lambda_{j} \in \mathbb{K}, b_{j} \in F, j=1, \ldots, m$.

When we have equality in (5), $t_{n}=1$. In this case we have a norm denoted by $\delta_{\left(r, \ldots, r_{n},+\infty\right)}$

3.4. Proposition. $\delta_{\left(s, r, \ldots, r_{n}+\infty\right)}$ is a $t_{n}$-norm on $E_{l} \otimes \ldots \otimes E_{n} \otimes F$ and $\varepsilon \leq \delta_{\left(s, r_{1}, \ldots, r_{n},+\infty\right)}$, where $\varepsilon$ denotes the injective norm on $E_{l} \otimes \ldots \otimes E_{n} \otimes F$.

3.5. Proposition. If $s \leq t, r_{k} \leq p_{k}, k=1, \ldots, n$ and

$$
\frac{1}{r_{1}}+\ldots+\frac{1}{r_{n}}-\frac{1}{s} \leq \frac{1}{p_{1}}+\ldots+\frac{1}{p_{n}}-\frac{1}{t}
$$

then 


$$
\delta_{\left(s, r_{1}, \ldots, r_{n},+\infty\right)} \leq \delta_{\left(t, p_{1}, \ldots, p_{n}+\infty\right)}
$$

Proof. It is an adaptation to this case of the proof of 2.5 .

3.6. Remark. It is a consequence of 3.5 . and the fact that $\delta_{(+\infty, \ldots,+\infty,+\infty)}$ $=\Pi$ (the projective norm) that all norms $\delta_{\left\{r, \ldots, r_{n},+\infty\right.}$ are reasonable crossnorms on $E_{1} \otimes \ldots \otimes E_{n} \otimes F$.

3.7. Theorem. The topological dual of $\left(E_{l} \otimes \ldots \otimes E_{n} \otimes F, \delta_{\left(s ;, r_{1}, \ldots, r,+\infty\right.}\right)$ is isometric to $\mathscr{S}_{a s}^{\left(s, r_{i}, \ldots, r_{n}\right)}\left(E_{l} \otimes \ldots \otimes E_{n} ; F^{\prime}\right)$.

Proof. (1) If $T \in \mathscr{S}_{a s}^{\left(s ; F_{i}, \ldots, r_{n}\right)}\left(E_{1} \otimes \ldots \otimes \mathrm{E}_{h} ; F^{\prime}\right)$ we consider the linear functional $f_{T}$ on $E_{1} \otimes \ldots \otimes E_{n} \otimes F$ defined by

$$
f_{T}(u)=\sum_{j=1}^{m} \lambda_{j} T\left(x_{1, j}, \ldots, x_{n, j}\right)\left(b_{j}\right)
$$

for $u$ with a representation of the form

$$
u=\sum_{j=1}^{m} \lambda_{j} x_{1, i} \otimes \ldots, x_{n, j} \otimes b_{j}
$$

where $m \in \mathbb{N}, \lambda_{j} \in \mathbb{K}, x_{k, j} \in E_{k}, k=1, \ldots, n, b_{j} \in F, j=1, \ldots, m$. Hence

$$
\begin{aligned}
& \left|f_{T}(u)\right| \leq\left\|\left(\lambda_{j}\right)_{j=1}^{m}\right\|_{s^{\prime}}\left\|\left(T\left(x_{1, j}, \ldots, x_{n j}\right)\right)_{j=1}^{m}\right\|_{s}\left\|\left(b_{j}\right)_{j=1}^{m}\right\|_{\infty} \\
& \leq\left\|\left(\lambda_{j}\right)_{j=1}^{m}\right\|_{s^{\prime}}\|T\|_{\left.a s, f s, r_{1}, \ldots, r_{n}\right)} \prod_{k=1}^{n}\left\|\left(x_{k_{j}}\right)_{j=1}^{m}\right\|_{w, r_{k}}\left\|\left(b_{j}\right)_{j=1}^{m}\right\|_{\infty}
\end{aligned}
$$

Thus $f_{T}$ is $\delta_{\left(s, r_{1}, \ldots, r_{4}+\infty\right)}$-continuous and 


$$
\left\|f_{T}\right\| \leq\|T\|_{a s ;\left(s, r_{1}, \ldots, r_{n}\right)}
$$

(2) Now we consider a continuous linear functional $f$ on $\left(E_{1} \otimes \ldots \otimes\right.$ $\left.E_{n} \otimes F, \delta_{(s, r, \ldots, r,+\infty \infty)}\right)$ and define the $n$-linear mapping $T_{f}$ from $E_{1} \times \ldots \times E_{n}$ into $F^{\prime}$ by

$$
T_{f}\left(x_{1}, \ldots x_{n}\right)(b)=f\left(x_{1} \otimes \ldots \otimes x_{n} \otimes b\right)
$$

for $x_{k} \in E_{k}, k=1, \ldots, n$ and $b \in F$.

We consider $m \in \mathbb{N}, x_{k, j} \in E_{k}, k=1, \ldots, n$ and $j=1, \ldots, m$. For each $\varepsilon>0$ there are $b_{j} \in F,\left\|b_{j}\right\|=1, j=1, \ldots, m$ such that

$$
\sum_{j=1}^{m}\left\|T_{f}\left(x_{1,}, \ldots, x_{n, j}\right)\right\|^{s} \leq \varepsilon+\sum_{j=1}^{m}\left|T_{f}\left(x_{1, j}, \ldots, x_{n, j}\right)(b j)\right|^{s}=\otimes
$$

For conveniente $\lambda_{j} \in \mathbb{R},\left|\lambda_{j}\right|=1, j=1, \ldots, m$ we may write

$$
\begin{aligned}
& \otimes=\varepsilon+\left|\sum_{j=1}^{m} \lambda_{j} f\left(\left|f\left(x_{1, j} \otimes \ldots \otimes x_{n, j} \otimes b_{j}\right)\right|^{s-1} x_{1, j} \otimes \ldots \otimes x_{n, j} \otimes b_{j}\right)\right| \\
& \leq \varepsilon+\|f\| \cdot \delta_{\left(s, r_{1}, \ldots, r_{n,}+\infty\right)}\left(\sum_{j=1}^{m} \lambda_{j}\left|f\left(x_{1, j} \otimes \ldots \otimes x_{n, j} \otimes b_{j}\right)\right|^{s-1} x_{1_{j}} \otimes \ldots \otimes x_{n, j} \otimes b_{j}\right) \\
& \leq \varepsilon+\|f\|\left[\sum_{j=1}^{m}\left|f\left(x_{1, j} \otimes \ldots \otimes x_{n j} \otimes b_{j}\right)\right|^{\left(s^{s-1}\right) s^{\prime}}\right]^{\frac{1}{s^{\prime}}} \prod_{k=1}^{n}\left\|\left(x_{k, j}\right)_{j=1}^{m}\right\|_{w, r_{k}}\left\|\left(b_{j}\right)_{j=1}^{m}\right\|_{\infty} \\
& \left.\leq \varepsilon+\|f\|\left[\| T_{f}\left(x_{1, j}, \ldots, x_{n j}\right)\right)_{j=1}^{m} \|_{s}\right]^{\frac{1}{s^{\prime}}} \prod_{k=1}^{n}\left\|\left(x_{k j}\right)_{j=1}^{m}\right\|_{k, r_{k}}
\end{aligned}
$$

Since $\varepsilon>0$ is arbitrary we have 


$$
\left\|\left(T_{f}\left(x_{1, j}, \ldots, x_{n_{j}}\right)\right)_{j=1}^{m}\right\|_{s} \leq i f f \prod_{k=1}^{n}\left\|\left(x_{k, j}\right)_{j=1}^{m}\right\|_{w, r_{k}}
$$

Hence $T_{f}$ is absolutely $\left(s ; r_{1}, \ldots, r_{n}\right)$-summing and

$$
\left\|T_{f}\right\|_{a s,\left(s, r_{1}, \ldots, r_{n}, r_{n}\right.} \leq\|f\|
$$

\section{CHARACTERIZATION OF THE TOPOLOGICAL DUALS OF THE SPACES OF MAPPINGS OF NUCLEAR TYPE}

In this section, $s, r_{k} \in[1,+\infty], k=1, \ldots, n$ and

$$
1 \leq \frac{1}{s}+\frac{1}{r_{1}^{\prime}}+\ldots+\frac{1}{r_{k}^{\prime}}
$$

If we consider $E_{1}^{\prime} \otimes \ldots \otimes E_{n}^{\prime} \otimes F$ with $\delta_{\left(s^{\prime} r^{\prime}, \ldots, r_{n,+\infty}\right)}$ it is clear that it is isometric to $\mathscr{L}_{f}\left(E_{1}, \ldots, E_{n} ; F\right)$ with $\|\cdot\|_{N_{\left(s ; r_{1}, \ldots, r_{n}\right)}}$ through the mapping taking

$$
u=\sum_{j=1}^{m} \lambda_{j} \varphi_{1, j} \otimes \ldots \otimes \varphi_{n, j} \otimes b_{j}
$$

into

$$
T_{u}=\sum_{j=1}^{m} \lambda_{j} \varphi_{1, j} \times \ldots \times \varphi_{n, j} b_{j}
$$

This fact, Theorem 3.7. and Theorem 2.10 give the following characterization result.

4.1. Theorem. If $E_{I}^{\prime}, \ldots, E_{n}^{\prime}$ have the bounded approximation property, the topological dual of $\mathscr{S}_{N}^{\left(s_{i}, r_{1}, \ldots, r_{n}\right)}\left(E_{l}, \ldots, E_{n} ; F\right)$ is isometric to

$\mathscr{L}_{a s}^{\left(s^{\prime} ; r_{1}^{\prime}, \ldots, r_{n}^{\prime}\right)}\left(E_{1}^{\prime}, \ldots, E_{n}^{\prime} ; F^{\prime}\right)$ through the mapping 


$$
B(\psi)\left(\varphi_{1}, \ldots, \varphi_{n}\right)(b)=\psi\left(\varphi_{1} \times \ldots \times \varphi_{n} b\right)
$$

for all $b \in F, \varphi_{k} \in E_{k}^{\prime}, k=1, \ldots, n$ and $\psi$ in the required dual.

In particular the topological dual of $\mathscr{S}_{N}^{\left(r_{1}, \ldots, r_{n}\right)}\left(E_{t}, \ldots, E_{n} ; F\right)$ is isometric to $\mathscr{P}_{d}^{\left(r^{\prime}, \ldots, r^{\prime}\right)}\left(E_{1}^{\prime}, \ldots, E_{n}^{\prime} ; F^{\prime}\right)[$ under condition (5) with $s \geq 1]$.

A result of A. Defant and J. Voigt (see [2] for a proof) state that $\mathscr{L}_{a s}\left(E_{1}, \ldots, E_{n} ; \mathbb{R}\right)$ is identically isometric to $\mathscr{L}\left(E_{1}, \ldots, E_{n} ; \mathbb{R}\right)$. If $n \geq 2$ it is proved in [2] that $\mathscr{L}_{a s}\left(E_{1}, \ldots, E_{n} ; F\right)$ is identically homeomorphic to $\mathscr{L}\left(E_{1}, \ldots, E_{n} ; F\right)$ when $E_{k}$ has the Orlicz property, $k=1, \ldots, n$. These results and Theorem 4.1 allow us to state the following results.

4.2. Theorem. If $E_{1}^{\prime}, \ldots, E_{n}$ have the bounded approximation property, the topological dual of the space of scalar valued n-linear mappings of nuclear type $(+\infty ;+\infty)$ on $E_{l} \times \ldots \times E_{n}$ is isometric to $\mathscr{L}\left(E_{l}, \ldots, E_{n}^{\prime}, \mathbb{K}\right)$.

4.3. Theorem. If $E_{k}$ is either $c_{0}$ or $\ell_{p}, p \in[2,+\infty)$, for $k=1, \ldots, n$ and $n \geq 2$, then the topological dual of $\mathscr{L}_{N}^{(+\infty ;+\infty)}\left(E_{1}, \ldots, E_{n} ; F\right)$ is homeomorphic to $\mathscr{P}\left(E_{i}, \ldots, E_{n} ; F^{\prime}\right)$.

Remark. When $s \leq r, s \in(0,+\infty]$ and $r \in[1,+\infty]$ it is possible to consider the $n$-homogeneous polynomials from $E$ into $F$ of nuclear type $(s ; r)$ canonically associated to the symmetric elements of $\mathscr{L}_{N}^{(s, r)}(E \ldots, E ; F)$. Analogous results as described in this paper are still valid for the spaces of polynomials. Now the use of the idea of homomorphy types (see [13] and [7]) allows us to consider holomorphic mappings of nuclear type $(s ; r)$ from open subsets of $E$ into $F$. See [10] for the case $s=r=1$. A careful lecture of the result of $[10]$ shows us that we can define a complete metrizable space of entire functions on $E$ of bounded nuclear type $(s ; r)$, with topological dual isomorphic to a natural subspace of "absolutely $\left(s^{\prime}, r^{\prime}\right)$-summing" entire functions on $E^{\prime}$. Again following the routine of [10] we can show that convolution equations on the space of entire functions of bounded nuclear type $(+\infty,+\infty)$ have always solutions and a 
Malgrange type approximation result holds for solutions of the homogeneous equations. Theorem 4.2. is a key lemma for the proof of these facts. A hard open problem is to know if results of this type for convolution equations can be proved when $(s ; r)$ is different from $(1 ; 1)$ and $(+\infty ;+\infty)$. Another problem is to check if the results of Dinnen's paper [7] are true in this case.

\section{References}

[1] ALENCAR, R.L. Multilinear mappings of nuclear type and integral type, Proc. A.M.S., 94 (1), 1985.

[2] ALENCAR, R.L. and MATOS, M.C. Some classes of multilinear mappings between Banach spaces. Publicaciones del Departamento de Análisis Matemático. Universidad Complutense de Madrid, 1989.

[3] ARON, R. Holomorphy types for open subsets of Banach spaces. Studia Math., 45 (1973), 273-289.

[4] BRAUNSS, H.A. Räume multilinear Abbildungen und deren Dualräume. Wiss. Zeitschr. Päd. Hochschule Potsdam, 28 (1984), 159-165.

[5] BOLAND, P.J. Malgrange theorem for entire functions on nuclear spaces. Proc. Infinite Dimensional Holomorphy. Lecture Notes in Mathematics, Ed. T.L. Hayden and T.J. Suffridge, 364 (1974), 135-144. Springer-Verlag.

[6] DINEEN, S. Complex Analysis in Locally Convex Spaces. Mathematics Studies, 57, Notas de Matemática 83, North-Holland, Amsterdam, 1981.

[7] DINEEN, S. Holomorphy types in a Banach space. Studia Math. 39 (1971), 241-288.

[8] DWYER III, T.A.W. Partial differential equations in Fisher-Fock spaces for the Hilbert-Schmidt holomorphy types. B.A.M.S., 77 (1971), 725-730.

[9] GROTHENDIECK, A. Sur une notion de produit tensorial topologique d' espaces vectoriels topologiques, et une class remarquable d' espaces vectoriels liées à cette notion. C.R. Acad. Sci. Paris 233 (1951), 1556-1558.

[10] GUPTA, C.P. Malgrange Theorem for nuclear entire functions of bounded type on a Banach space. Notas de Matemática 37 (1968) and Indag. Math. 32 (1970), 356-358.

[11] MATOS, M.C. On holomorphy in Banach spaces and absolute convergence of Fourier series. Portugaliae Mathematica 45 (4) (1988), 429-450. 
[12] MATOS, M.C. and NACHBIN, L. Silva holomorphy types. Functional Analysis, Holomorphy and Approximation Theory. Ed. S. Machado. Lecture Notes in Math. 843 (1981), Springer-Velar, 437-487.

[13] NACHBIN, L. Topology on spaces of holomorphic mappings. Ergebn. Math. Grenzgeb. 47. Springer-Verlag, New York, 1969.

[14] NACHBIN, L. Concerning holomorphy types for Banach spaces. Int. Coll. Nuclear Spaces and Ideals in Operators Algebras. Studia Math. 38 (1970), 407412.

[15] PIETSCH, A. Ideals of Multilinear Functionals. Proc. II Intem. Conf. on Operator Algebras, Ideals and Appl. in Theoretical Physics. Leipzig (1983) Teubner-Texte.

[16] PIETSCH, A. Operator Ideals. Dt. Verl. Wissenschafter, Berlin (1978) and North-Holland, Amsterdam (1980).

[17] RUSTON, A.F. Direct products of Banach spaces and linear functional equations. Proc. London Math. Soc. (3) 1 (1951), 327-348.

[18] RUSTON, A.F. On the Fredholm theory of integral equations for operators belonging to the trace class of a general Banach space. Proc. London Math. Soc. (2) 53 (1951), 109-124.

[19] SCHNEIDER, B. On absolutely p-summing and related multilinear mappings. Wiss. Zeitschr. Brandenb. Landeshochschule Potsdam, 35 (1991), 105-117.

IMECC-UNICAMP

Caixa Postal 6065

13081 Campinas, S.P.

BRASIL
Recibido: 13 de noviembre de 1991 Revisado: 15 de octubre de 1992 